Article

\title{
Application of Boroisoquinoline Fluorophores as Chemodosimeters for Fluoride Ion and Pd (0)
}

\author{
Dénes Sóvári, György Miklós Keserú and Péter Ábrányi-Balogh *(D) \\ Research Centre for Natural Sciences, Institute of Organic Chemistry, Medicinal Chemistry Research Group, \\ POB 286, 1519 Budapest, Hungary; sovari.denes@ttk.hu (D.S.); keseru.gyorgy@ttk.hu (G.M.K.) \\ * Correspondence: abranyi-balogh.peter@ttk.hu; Tel.: +36-1-382-6961
}

Received: 12 November 2019; Accepted: 26 December 2019; Published: 2 January 2020

\begin{abstract}
The development of novel chemodosimeters is currently a prosperous field in organic chemistry. Recently, a new family of fluorophores, the boroisoquinolines, were introduced with satisfying photophysical properties. As a continuation of this research, the application of boroisoquinolines is presented as chemodosimeters for fluoride anion and $\mathrm{Pd}(0)$. The new tools showed good selectivity for the detection of the analytes. Moreover, the mechanism of action was investigated experimentally.
\end{abstract}

Keywords: boroisoquinolines; chemodosimeter; fluorescence; fluorescent molecular probe; fluoride; palladium (0)

\section{Introduction}

Chemodosimeters and chemosensors are handy chemical tools for the detection of ions, metals, or reactive species. In most cases, it is prominently significant that the analyte has biological activity or at least some effect on living organisms [1,2]. The mechanism of action of the chemodosimeters is usually based on an irreversible chemical reaction with the analyte that changes the structure of the probe, and causes a well-sensible shift in fluorescence properties. This might be an on/off signal (turn-on and turn-off probes) [3]. The first fluoride chemodosimeter was published in 2000 [4,5], while the first compound that was able to detect Pd (0) besides $\mathrm{Pd}^{2+}$ was reported in 2007 [6]. Notably, since then, the number of fluorescent molecular probes have been rising rapidly [7] due to the needs of fast and on-the-spot analytical testing that turned inevitable the development of this frontier field of organic and analytical chemistry.

Although fluoride anions are elemental for bone and tooth health, it was recognized that the presence of fluoride in a larger amount might cause skeletal fluorosis, calcification, and weakening of the bone tissue [8-10]. Thus, in rural areas of developing countries, where one of the main water-polluting component is the fluoride anion [1], it became important to find analytical tools for its recognition. This induced the development of various fluoride fluorescent probes: reversible and irreversible ones (1-3) as well. The mechanism of the reversible compounds rests on salt formation [11,12], while the irreversibly acting substances contain generally a $\mathrm{C}-\mathrm{Si}(\mathbf{1})$ or $\mathrm{O}-\mathrm{Si}(\mathbf{2}, \mathbf{3})$ bond as a recognition site (Figure 1), and take advantage of the high reactivity of fluoride towards silicon [13-16]. There are five other types of fluorescens fluoride test compounds: organoboron compounds, nucleophilic addition based probes, intramolecular hydrogen transfer reaction based agents, metal-mediated probes, and bifunctional fluorescent test compounds [1]. 


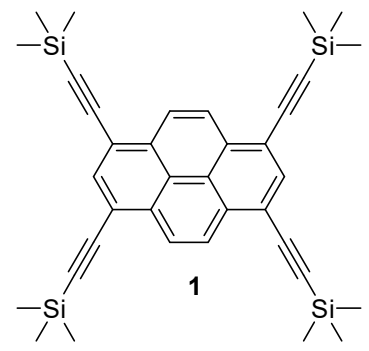

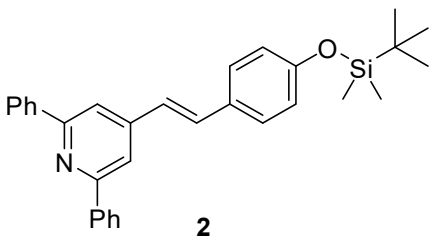

2

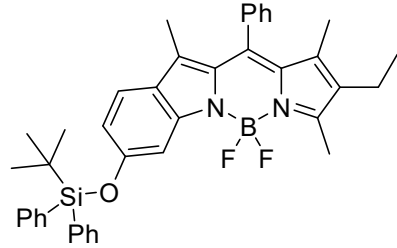

Figure 1. Irreversible fluoride-selective fluorescent probes.

Palladium is widely used in transition metal-catalyzed reactions and for reductions in chemical research and industry as well as in the catalysators of exhaust-pipes in vehicles [17]. The emerging number of cars with palladium containing catalyst results in the raise of the palladium-level in the air causing allergic symptoms and other maleficent biological effects when getting into living organisms [2]. The Pd (0) chemodosimeters are usually based on catalytic processes. In most cases, the fluorophore skeleton is equipped with an $O$-allyl or $O$-propargyl group, which is removed by the palladium provoking the sensible photophysical change [17-19]. The photospectroscopic properties might change due to a ring closure by intramolecular acylation (4), or the by the removal of the O-propargyl (5) or the O-(O-allyl) carboxyl group (6), which turns on the ESIPT (Excited State Intramolecular Proton Transfer) effect (Figure 2).<smiles>C#CCOc1ccc2ccccc2c1C=C(C#N)C#N</smiles>

4<smiles>C#CCOc1cccc2c1C(=O)N(CC)C2C(C)COCCOCC</smiles><smiles>C=CCOC(=O)Oc1ccc(/C=C/C2=CC(=C(C#N)C#N)c3ccccc3O2)cc1</smiles>

Figure 2. Palladium chemodosimeters.

Recently, we developed a new fluorescent compound family, namely the boroisoquinolines (Figure 3, BIQ), showing good photophysical properties [20]. As the continuation of our research, we aimed to synthetize derivatives for analytical applications, in particular for the detection of fluoride (F-BIQ) and palladium (Pd-BIQ), as a development of chemodosimeters based on the novel fluorophores (Figure 3). We envisaged that the recognition elements would be the tert-butyldiphenylsilyl group for the fluoride anion, and the $O$-allyl moiety for $\mathrm{Pd}(0)$ sensing.

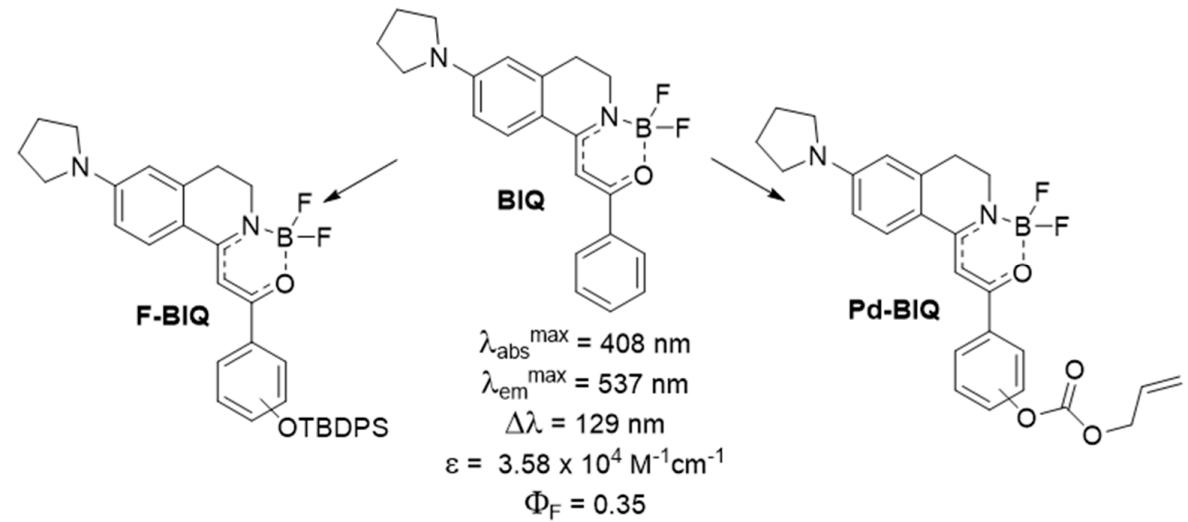

Figure 3. Member of the boroisoquinoline family (BIQ) and the proposed chemodosimeters (F-BIQ, Pd-BIQ). 


\section{Materials and Methods}

All melting points were determined on a Jasco SRS OptiMelt apparatus (Stanford, CA, USA) and are uncorrected. ${ }^{1} \mathrm{H}$ and ${ }^{13} \mathrm{C}$ NMR spectra were recorded in $\mathrm{CDCl}_{3}$ or DMSO- $d_{6}$ solution at room temperature, on a Varian Unity Inova 500 spectrometer $\left(500\right.$ and $125 \mathrm{MHz}$ for ${ }^{1} \mathrm{H}$ and APT NMR spectra, respectively), and on a Varian Unity Inova 300 spectrometer $(300,75 \mathrm{MHz}$ and $282 \mathrm{MHz}$ for ${ }^{1} \mathrm{H}$, APT NMR and ${ }^{19} \mathrm{~F}$ NMR spectra, respectively), with the deuterium signal of the solvent as the lock and TMS as the internal standard. Chemical shifts $(\delta)$ and coupling constants $(J)$ are given in ppm and $\mathrm{Hz}$, respectively. High resolution mass spectra were recorded on a Waters Q-TOF Premier mass spectrometer in positive ESI ionization mode. The reactions were followed by analytical thin layer chromatography on silica gel $60 \mathrm{~F}_{254}$ and HPLC-MS chromatography with a Shimadzu LCMS-2020 device using a Reprospher $100 \mathrm{C} 18(5 \mu \mathrm{m} ; 100 \times 3 \mathrm{~mm})$ column and positive-negative double ion source (DUIS) with a quadrupole MS analysator in a range of 50-1000 m/z. All reagents were purchased from commercial sources [e.g., Sigma Aldrich (St. Louis, MO, USA), Fluorochem (Hadfield, Derbyshire, UK), Alfa (Haverhill, MA, USA), Combi-Blocks (San Diego, CA, USA)]. Analytical samples of new compounds were obtained by trituration or recrystallization from the solvents or solvent mixtures given below in parentheses.

\subsection{Photophysical Measurements}

The fluorescence and absorbance measurements were carried out on a Jasco FP8300 spectrofluorometer, in a standard cell quartz cuvette with $1 \mathrm{~cm}$ light path length. The widths of the excitation slit and the emission slit were both set to $2.5 \mathrm{~nm}$ with the scanning speed at $1000 \mathrm{~nm} / \mathrm{min}$. Pure solvents were used as blank correction. We used acetonitrile as solvent if not otherwise mentioned, and the concentration was $1 \mu \mathrm{M}$. For water tests, we used $10 \mu \mathrm{M}$ solutions of the chemodosimeters, made with $\mathrm{pH}=7.4$ PBS (phosphate buffered saline) buffer with $0.1 \%$ SDS (sodium dodecyl sulfate). We measured the absorbance spectra from $250 \mathrm{~nm}$ to $500 \mathrm{~nm}$. The excitement spectra were recorded between $250 \mathrm{~nm}$ and $500 \mathrm{~nm}$. For the emission spectra, we excited the sample at the excitation maximum, and recorded it from excitation maximum plus $10 \mathrm{~nm}$ to $800 \mathrm{~nm}$. The test solutions were made in PBS puffer ( $50 \mathrm{mM}$ phosphate, $\mathrm{pH}=7.4$ ) for aqueous measurements, or in acetonitrile. For the investigation of the 12a chemodosimeter, we used tetrabutylammonium (TBA) salts, and, for the 12d chemodosimeter, we used chloride salts and tri (dibenzylideneacetone) dipalladium (0) $\left(\mathrm{Pd}_{2} \mathrm{dba}_{3}\right)$.

\subsection{Synthetic Procedures}

\subsubsection{General Procedure for the Preparation of 1-Methylidene-3,4-Dihydroisoquinolines (11)}

In a three-necked round bottom flask $20 \mathrm{~mL}$ of anhydrous tetrahydrofuran and $0.42 \mathrm{~mL}$ of diisopropylamine $(0.30 \mathrm{~g}, 3.0 \mathrm{mmol}, 1.2$ equiv. $)$ were cooled below $-70{ }^{\circ} \mathrm{C}$, in a nitrogen atmosphere. Then, $3 \mathrm{mmol}$ (1.2 equiv.) $n$-BuLi was added dropwise, and the reaction mixture was stirred for $1 \mathrm{~h}$. Then, $2.5 \mathrm{mmol}$ isoquinoline (9) in tetrahydrofuran solution $(0.54 \mathrm{~g})$ was added dropwise. The reaction mixture was stirred for $1 \mathrm{~h}$, then the appropriate ester ( $3.0 \mathrm{mmol}, 1.2$ equiv.) was added dropwise in $10 \mathrm{~mL}$ tetrahydrofuran [11a: ethyl 4-((tert-butyldiphenylsilyl) oxy) benzoate (10a) (2.5 g, 2.5 equiv.); $\mathbf{1 1 b}$ ethyl-4-methoxybenzoate (10b) (1.1 g, 2.5 equiv.)]. Then, the reaction mixture was allowed to warm to room temperature and stirred for $1 \mathrm{~h}$. The solution was quenched with $30 \mathrm{~mL}$ water, and extracted with $80 \mathrm{~mL}$ ethyl acetate. The aqueous layer was washed with ethyl acetate $(2 \times 30 \mathrm{~mL})$ and dichloromethane $(2 \times 30 \mathrm{~mL})$. The combined organic phases were dried $\left(\mathrm{MgSO}_{4}\right)$, filtered and evaporated under reduced pressure. The residue was purified by flash column chromatography on aluminum oxide using hexane/ethyl acetate.

(Z)-1-(4-((tert-butyldiphenylsilyl) oxy) phenyl)-2-(6-(pyrrolidin-1-yl)-3,4-dihydroisoquinolin-1(2H)-ylidene) ethanone (11a). Yield: $2.1 \mathrm{~g}(85 \%)$, yellow crystals, m.p. $66-68{ }^{\circ} \mathrm{C}\left(\mathrm{CDCl}_{3}\right) ;{ }^{1} \mathrm{H} \mathrm{NMR}\left(300 \mathrm{MHz}, \mathrm{CDCl}_{3}\right)$ $\delta 11.68(\mathrm{~s}, 1 \mathrm{H}, \mathrm{NH}), 7.75-7.73(\mathrm{~m}, 2 \mathrm{H}, \mathrm{ArH}), 7.73-7.71(\mathrm{~m}, 4 \mathrm{H}, \mathrm{ArH}), 7.62(\mathrm{~d}, J=8.8 \mathrm{~Hz}, 1 \mathrm{H}, \mathrm{ArH})$, 7.45-7.40 (m, 2H, ArH), $7.37(\mathrm{t}, J=7.3 \mathrm{~Hz}, 4 \mathrm{H}, \mathrm{ArH}), 6.79(\mathrm{~d}, J=8.6 \mathrm{~Hz}, 2 \mathrm{H}, \mathrm{ArH}), 6.44(\mathrm{dd}, J=8.8,2.4$ 
$\mathrm{Hz}, 1 \mathrm{H}, \mathrm{ArH}), 6.30(\mathrm{~d}, J=2.0 \mathrm{~Hz}, 1 \mathrm{H}, \mathrm{ArH}), 6.12(\mathrm{~s}, 1 \mathrm{H}, \mathrm{HC}=), 3.49\left(\mathrm{t}, J=5.7 \mathrm{~Hz}, 2 \mathrm{H}, \mathrm{CH}_{2}\right), 3.33(\mathrm{t}$, $\left.J=6.5 \mathrm{~Hz}, 4 \mathrm{H}, \mathrm{CH}_{2}\right), 2.86\left(\mathrm{t}, J=6.5 \mathrm{~Hz}, 2 \mathrm{H}, \mathrm{CH}_{2}\right), 2.05-1.99\left(\mathrm{~m}, 4 \mathrm{H}, \mathrm{CH}_{2}\right), 1.12\left(\mathrm{~s}, 9 \mathrm{H}, \mathrm{CH}_{3}\right) \mathrm{ppm} ;{ }^{13} \mathrm{H}$ NMR $\left(125 \mathrm{MHz}, \mathrm{CDCl}_{3}\right) \delta 186.6(\mathrm{C}=\mathrm{O}), 159.4(\mathrm{C}=), 157.4(\mathrm{C}=), 149.7(\mathrm{C}=), 138.3(\mathrm{C}=), 135.5(=\mathrm{CH})$, $134.7(\mathrm{C}=), 132.8(\mathrm{C}=), 129.9(=\mathrm{CH}), 128.3(=\mathrm{CH}), 127.8(=\mathrm{CH}), 127.3(=\mathrm{CH}), 119.2(=\mathrm{CH}), 116.2(\mathrm{C}=)$, $110.21(=\mathrm{CH}), 84.9(=\mathrm{CH}), 47.5\left(\mathrm{CH}_{2}\right), 38.8\left(\mathrm{CH}_{2}\right), 29.1\left(\mathrm{CH}_{2}\right), 26.5\left(\mathrm{CH}_{3}\right), 25.4\left(\mathrm{CH}_{2}\right), 19.5(\mathrm{C}=)$ ppm; $[\mathrm{M}+\mathrm{H}]^{+}$measured $=573.2917$, calcd. for $\mathrm{C}_{37} \mathrm{H}_{41} \mathrm{~N}_{2} \mathrm{O}_{2}$ Si: 573.2931 .

(Z)-1-(4-methoxyphenyl)-2-(6-(pyrrolidin-1-yl)-3,4-dihydroisoquinolin-1(2H)-ylidene) ethanone (11b). Yield: $0,43 \mathrm{~g}(40 \%)$, yellow crystals, m.p. $186{ }^{\circ} \mathrm{C}$ (hexane-ethyl acetate); ${ }^{1} \mathrm{H}$ NMR $\left(500 \mathrm{MHz}, \mathrm{CDCl}_{3}\right) \delta$ $11.72(\mathrm{~s}, 1 \mathrm{H}, \mathrm{NH}), 7.96-7,87(\mathrm{~m}, 2 \mathrm{H}, \mathrm{ArH}), 7,67$ (d, $J=8,8 \mathrm{~Hz}, 1 \mathrm{H}, \mathrm{ArH}), 6,93-6,89(\mathrm{~m}, 2 \mathrm{H}, \mathrm{ArH}), 6,46$ $(\mathrm{dd}, J=8,7,2,4 \mathrm{~Hz}, 1 \mathrm{H}, \mathrm{ArH}), 6,31(\mathrm{~d}, J=2,2 \mathrm{~Hz}, 1 \mathrm{H}, \mathrm{ArH}), 6,19(\mathrm{~s}, 1 \mathrm{H}, \mathrm{HC}=), 3,84\left(\mathrm{~s}, 3 \mathrm{H}, \mathrm{OCH}_{3}\right), 3,50$ $\left(\mathrm{tt}, J=8,6,4,3 \mathrm{~Hz}, 2 \mathrm{H}, \mathrm{CH}_{2}\right), 3,33\left(\mathrm{t}, J=6,6 \mathrm{~Hz}, 4 \mathrm{H}, \mathrm{CH}_{2}\right), 2,88\left(\mathrm{t}, J=6,6 \mathrm{~Hz}, 2 \mathrm{H}, \mathrm{CH}_{2}\right), 2,07-1,97(\mathrm{~m}$, $\left.4 \mathrm{H}, \mathrm{CH}_{2}\right) \mathrm{ppm} ;{ }^{13} \mathrm{C} \mathrm{NMR}\left(125 \mathrm{MHz}, \mathrm{CDCl}_{3}\right) \delta 186,5(\mathrm{C}=\mathrm{O}), 160,4(\mathrm{C}=\mathrm{O}), 153,5(\mathrm{C}=), 152,6(\mathrm{C}=), 150,1$ $(\mathrm{CH}=), 139,9(\mathrm{C}=), 138,7(\mathrm{CH}=), 131,4(\mathrm{C}=), 128,4(\mathrm{CH}=), 127,7(=\mathrm{CH}), 120,8(=\mathrm{CH}), 119,8(\mathrm{C}=), 116,0$ $(\mathrm{C}=), 110,6(=\mathrm{CH}), 110,5(=\mathrm{CH}), 85,5\left(=\mathrm{CH}_{2}\right), 69,4\left(\mathrm{CH}_{2}\right), 47,8\left(\mathrm{CH}_{2}\right), 39,1\left(\mathrm{CH}_{2}\right), 29,2\left(\mathrm{CH}_{2}\right), 25,7\left(\mathrm{CH}_{2}\right)$ ppm; $[\mathrm{M}+\mathrm{H}]^{+}$measured $=349.1908$, calcd. for $\mathrm{C}_{22} \mathrm{H}_{25} \mathrm{~N}_{2} \mathrm{O}_{2}: 349.1910$.

(Z)-allyl (4-(2-(6-(pyrrolidin-1-yl)-3,4-dihydroisoquinolin-1(2H)-ylidene) acetyl) phenyl) carbonate (11d).

In a round bottom flask, $0.87 \mathrm{~g}$ (Z)-1-(4-methoxyphenyl)-2-(6-(pyrrolidin-1-yl)-3, 4-dihydroisoquinolin-1 (2H)-ylidene) ethanone (11b) (2.5 mmol) was dissolved in $25 \mathrm{~mL}$ anhydrous dichloromethane. The flask was flushed with argon and cooled to $0{ }^{\circ} \mathrm{C}$, then $7.7 \mathrm{~mL} 1 \mathrm{M}$ $\mathrm{BBr}_{3}$ dichloromethane solution $(7.5 \mathrm{mmol}, 3$ equiv.) was added dropwise. The reaction mixture was stirred at room temperature overnight. The next day it was quenched with $20 \mathrm{~mL}$ water; then, the water layer was extracted with dichloromethane ten times. The combined organic layers were dried $\left(\mathrm{MgSO}_{4}\right)$, filtered, and concentrated in vacuo. Then, the residue was dissolved in $60 \mathrm{~mL}$ anhydrous tetrahydrofuran and $50 \mathrm{~mL}$ dichloromethane, $0.49 \mathrm{~mL}$ triethylamine $(0.35 \mathrm{~g}, 3.5 \mathrm{mmol}, 1.4$ equiv. $)$ was added and the mixture was cooled below $-10^{\circ} \mathrm{C}$. In addition, $0.32 \mathrm{~mL}$ allyl chloroformate $(0.36 \mathrm{~g}$, $3 \mathrm{mmol}, 1.2$ equiv.) was added dropwise; then, the reaction mixture was let to warm room temperature and stirred overnight. The next day the reaction mixture was reduced in vacuo. The residue was purified by flash chromatography on silica gel with hexane/ethyl acetate.

Yield: $0.16 \mathrm{~g}(15 \%)$, brown film; ${ }^{1} \mathrm{H}$ NMR $\left(300 \mathrm{MHz}, \mathrm{CDCl}_{3}\right) \delta 11.79(\mathrm{~s}, 1 \mathrm{H}, \mathrm{NH}), 7.95(\mathrm{~d}, J=8.3 \mathrm{~Hz}$, $2 \mathrm{H}, \mathrm{ArH}), 7.66(\mathrm{~d}, J=8.7 \mathrm{~Hz}, 1 \mathrm{H}, \mathrm{ArH}), 7.22(\mathrm{~d}, J=8.3 \mathrm{~Hz}, 2 \mathrm{H}, \mathrm{ArH}), 6.47(\mathrm{~d}, J=8.6 \mathrm{~Hz}, 1 \mathrm{H}, \mathrm{ArH}), 6.32$ $(\mathrm{s}, 1 \mathrm{H}, \mathrm{ArH}), 6.17(\mathrm{~s}, 1 \mathrm{H}, \mathrm{HC}=), 6.01(\mathrm{dq}, J=10.6,5.7 \mathrm{~Hz}, 1 \mathrm{H}, \mathrm{HC}=), 5.43(\mathrm{~d}, J=17.1 \mathrm{~Hz}, 1 \mathrm{H}, \mathrm{HC}=), 5.33$ $(\mathrm{d}, J=10.4 \mathrm{~Hz}, 1 \mathrm{H}, \mathrm{HC}=), 4.75\left(\mathrm{~d}, J=5.7 \mathrm{~Hz}, 2 \mathrm{H}, \mathrm{CH}_{2}\right), 3.52\left(\mathrm{~s}, 2 \mathrm{H}, \mathrm{CH}_{2}\right), 3.35\left(\mathrm{~s}, 4 \mathrm{H}, \mathrm{CH}_{2}\right), 2.89(\mathrm{t}$, $\left.J=6.2 \mathrm{~Hz}, 2 \mathrm{H}, \mathrm{CH}_{2}\right), 2.03\left(\mathrm{~s}, 4 \mathrm{H}, \mathrm{CH}_{2}\right) \mathrm{ppm} ;{ }^{13} \mathrm{C} \mathrm{NMR}\left(75 \mathrm{MHz}, \mathrm{CDCl}_{3}\right) \delta 186.0(\mathrm{C}=\mathrm{O}), 160.4(\mathrm{C}=\mathrm{O})$, $153.5(\mathrm{C}=), 152.6(\mathrm{CH}=), 150.1(\mathrm{CH}=), 139.6(\mathrm{CH}=), 138.7(\mathrm{CH}=), 131.4(\mathrm{C}=), 128.4(\mathrm{CH}=), 127.7(\mathrm{C}=)$, $120.8(\mathrm{CH}=), 119.8(\mathrm{C}=), 116.0(\mathrm{C}=), 110.6(\mathrm{C}=), 110.5(\mathrm{CH}=), 85,5\left(\mathrm{CH}_{2}\right), 69.4\left(\mathrm{CH}_{2}\right), 47.8\left(\mathrm{CH}_{2}\right), 39.1$ $\left(\mathrm{CH}_{2}\right), 29.2\left(\mathrm{CH}_{2}\right), 25.7\left(\mathrm{CH}_{2}\right)$ ppm; $[\mathrm{M}+\mathrm{H}]^{+}{ }_{\text {measured }}=419.1984$, calcd. for $\mathrm{C}_{25} \mathrm{H}_{27} \mathrm{~N}_{2} \mathrm{O}_{4}: 419.1970$.

\subsubsection{General Procedure for the Preparation of Boroisoquinolines (12)}

Into a round bottom flask, $1 \mathrm{mmol}$ appropriate 1-methylidene-3,4-dihydroisoquinoline (14) was measured \{(Z)-1-(4-((tert-butyldiphenylsilyl) oxy) phenyl)-2-(6-(pyrrolidin-1-yl)-3, 4-dihydroisoquinolin-1 (2H)-ylidene) ethanone (11a) (0.57 g); ((Z)-allyl (4-(2-(6-(pyrrolidin-1-yl)-3, 4-dihydroisoquinolin-1 (2H)-ylidene) acetyl) phenyl) carbonate (11d) (0.42 g)\}, and dissolved in $5 \mathrm{~mL}$ dichloromethane. The solution was cooled to $0{ }^{\circ} \mathrm{C}, 0.91 \mathrm{~mL}$ diisopropylethylamine $(0.65 \mathrm{~g}, 5.0 \mathrm{mmol}, 5.0$ equiv) was added. $0.64 \mathrm{~mL}$ trifluorborane diethyl etherate $(0.71 \mathrm{~g}, 5.0 \mathrm{mmol}, 5.0$ equiv $)$ was added dropwise. The reaction mixture was stirred for $30 \mathrm{~min}$ at room temperature. The reaction mixture was concentrated and purified by flash column chromatography on silica gel using hexane/dichloromethane.

(Z)-1-(4-((tert-butyldiphenylsilyl) oxy) phenyl)-2-(2-(difluoroboranyl)-6-(pyrrolidin-1-yl)-3, 4-dihydroisoquinolin-1 (2H)-ylidene) ethan-1-one (12a).

Yield: $0.33 \mathrm{~g}(53 \%)$, yellow crystals, m.p. $261-262{ }^{\circ} \mathrm{C}$ (hexane-dichloromethane); ${ }^{1} \mathrm{H}$ NMR $\left(500 \mathrm{MHz}, \mathrm{CDCl}_{3}\right) \delta 7.74(\mathrm{t}, J=5.9 \mathrm{~Hz}, 2 \mathrm{H}, \mathrm{ArH}), 7.73-7.69(\mathrm{~m}, 4 \mathrm{H}, \mathrm{ArH}), 7.58(\mathrm{~d}, J=8.8 \mathrm{~Hz}, 1 \mathrm{H}, \mathrm{ArH})$, 
$7.44(\mathrm{dd}, J=8.4,6.3 \mathrm{~Hz}, 2 \mathrm{H}, \mathrm{ArH}), 7.37(\mathrm{t}, J=7.3 \mathrm{~Hz}, 4 \mathrm{H}, \mathrm{ArH}), 6.79(\mathrm{~d}, J=8.8 \mathrm{~Hz}, 2 \mathrm{H}, \mathrm{ArH}), 6.45(\mathrm{dd}$, $J=8.8,2.3 \mathrm{~Hz}, 1 \mathrm{H}, \mathrm{HC}=), 6.33(\mathrm{~s}, 2 \mathrm{H}, \mathrm{ArH}), 3.71\left(\mathrm{t}, J=6.9 \mathrm{~Hz}, 2 \mathrm{H}, \mathrm{CH}_{2}\right), 3.37\left(\mathrm{t}, J=6.5 \mathrm{~Hz}, 4 \mathrm{H}, \mathrm{CH}_{2}\right)$, $2.93-2.82\left(\mathrm{~m}, 2 \mathrm{H}, \mathrm{CH}_{2}\right), 2.04\left(\mathrm{dd}, J=7.9,5.2 \mathrm{~Hz}, 4 \mathrm{H}, \mathrm{CH}_{2}\right), 1.12\left(\mathrm{~s}, 9 \mathrm{H}, \mathrm{CH}_{3}\right) \mathrm{ppm} ;{ }^{13} \mathrm{C} \mathrm{NMR}(125$ $\left.\mathrm{MHz}, \mathrm{CDCl}_{3}\right) \delta 189.2(\mathrm{C}=\mathrm{O}), 174.0(\mathrm{C}=), 170.1(\mathrm{C}=), 167.5(\mathrm{C}=), 161.6(\mathrm{C}=), 158.6(\mathrm{C}=), 151.0(\mathrm{C}=)$, $139.8(\mathrm{C}=), 135.4(\mathrm{C}=), 132.4(=\mathrm{CH}), 130.1(=\mathrm{CH}), 129.1(=\mathrm{CH}), 128.5(=\mathrm{CH}), 127.9(=\mathrm{CH}), 119.7(=\mathrm{CH})$, 114.2 $(\mathrm{C}=), 110.3(=\mathrm{CH}), 110.1(=\mathrm{CH}), 88.5(=\mathrm{CH}), 47.6\left(\mathrm{CH}_{2}\right), 40,4\left(\mathrm{CH}_{2}\right), 27.9\left(\mathrm{CH}_{2}\right), 26.5\left(\mathrm{CH}_{3}\right), 25.4$ $\left(\mathrm{CH}_{2}\right), 19.5\left(\mathrm{CH}_{3}\right) \mathrm{ppm} ;{ }^{19} \mathrm{~F}$ NMR $\left(282 \mathrm{MHz}, \mathrm{CDCl}_{3}\right) \delta-140.63--140.75(\mathrm{~m}, 1 \mathrm{~F}, \mathrm{BF}),-140.75--140.87$ $(\mathrm{m}, 1 \mathrm{~F}, \mathrm{BF})$.; $[\mathrm{M}-\mathrm{F}]^{+}$measured $=601.2875$, calcd. for $\mathrm{C}_{37} \mathrm{H}_{39} \mathrm{BN}_{2} \mathrm{O}_{2} \mathrm{~F}: 601.2852$.

(Z)-allyl (4-(2-(2-(difluoroboranyl)-6-(pyrrolidin-1-yl)-3, 4-dihydroisoquinolin-1 (2H)-ylidene) acetyl) phenyl) carbonate (12d).

Yield: $0.34 \mathrm{~g}(72 \%)$, yellow crystals, m.p. $180{ }^{\circ} \mathrm{C}$ (hexane-dichloromethane); ${ }^{1} \mathrm{H}$ NMR $(300 \mathrm{MHz}$, $\left.\mathrm{CDCl}_{3}\right) \delta 7.99(\mathrm{~d}, J=7.5 \mathrm{~Hz}, 2 \mathrm{H}, \mathrm{NH}), 7.64(\mathrm{~d}, J=8.7 \mathrm{~Hz}, 1 \mathrm{H}, \mathrm{ArH}), 7.27(\mathrm{~d}, J=7.1 \mathrm{~Hz}, 2 \mathrm{H}, \mathrm{ArH}), 6.48$ $(\mathrm{d}, J=11.8 \mathrm{~Hz}, 2 \mathrm{H}, \mathrm{ArH}), 6.35(\mathrm{~s}, 1 \mathrm{H}, \mathrm{HC}=), 6.01(\mathrm{dq}, J=10.2,5.9 \mathrm{~Hz}, 1 \mathrm{H}, \mathrm{HC}=), 5.44(\mathrm{~d}, J=17.1 \mathrm{~Hz}$, $1 \mathrm{H}, \mathrm{HC}=), 5.34(\mathrm{~d}, J=10.4 \mathrm{~Hz}, 1 \mathrm{H}, \mathrm{HC}=), 4.75\left(\mathrm{~d}, J=5.7 \mathrm{~Hz}, 2 \mathrm{H}, \mathrm{CH}_{2}\right), 3.75\left(\mathrm{t}, J=6.4 \mathrm{~Hz}, 2 \mathrm{H}, \mathrm{CH}_{2}\right)$, $3.44-3.34\left(\mathrm{~m}, 4 \mathrm{H}, \mathrm{CH}_{2}\right), 2.91\left(\mathrm{t}, J=6.6 \mathrm{~Hz}, 2 \mathrm{H}, \mathrm{CH}_{2}\right), 2.11-2.01\left(\mathrm{~m}, 4 \mathrm{H}, \mathrm{CH}_{2}\right) ;{ }^{13} \mathrm{C} \mathrm{NMR}(75 \mathrm{MHz}$, $\left.\mathrm{CDCl}_{3}\right) \delta 186.0(\mathrm{C}=\mathrm{O}), 160.4(\mathrm{C}=\mathrm{O}), 153.5(\mathrm{C}=), 152.6(\mathrm{C}=), 150.1(\mathrm{CH}=), 139.9(\mathrm{CH}=), 138.7(\mathrm{C}=)$, 131.4 $(\mathrm{C}=), 128.4(\mathrm{CH}=), 127.7(\mathrm{CH}=), 120.8(\mathrm{CH}=), 119.8(\mathrm{C}=), 116.0(\mathrm{C}=), 110.6(\mathrm{CH}=), 110.5(\mathrm{CH}=)$, 85.5 $\left(\mathrm{CH}_{2}\right), 69.4\left(\mathrm{CH}_{2}\right), 47.8\left(\mathrm{CH}_{2}\right), 39.1\left(\mathrm{CH}_{2}\right), 29.2\left(\mathrm{CH}_{2}\right), 25.7\left(\mathrm{CH}_{2}\right) \mathrm{ppm} ;{ }^{19} \mathrm{~F} \mathrm{NMR}\left(282 \mathrm{MHz}, \mathrm{CDCl}_{3}\right)$ $\delta-140.67--140.92(\mathrm{~m}, 1 \mathrm{~F}, \mathrm{BF}),-140.93--141.19(\mathrm{~m}, 1 \mathrm{~F}, \mathrm{BF}) .,[\mathrm{M}-\mathrm{F}]^{+}$measured $=447.1909$, calcd. for $\mathrm{C}_{25} \mathrm{H}_{25} \mathrm{BN}_{2} \mathrm{O}_{4} \mathrm{~F}: 447.1885$.

(Z)-2-(2-(difluoroboranyl)-6-(pyrrolidin-1-yl)-3,4-dihydroisoquinolin-1 (2H)-ylidene)-1-(4-hydroxyphenyl) ethan-1-one (12c).

In a round bottom flask, $0.06 \mathrm{~g}(Z)-1-(4-($ tert-butyldiphenylsilyl) oxy) phenyl)-2-(2-(difluoroboranyl)6-(pyrrolidin-1-yl)-3, 4-dihydroisoquinolin-1 (2H)-ylidene) ethan-1-one (15a) $(0.1 \mathrm{mmol})$ was dissolved in HPLC grade acetonitrile. Then, $0.32 \mathrm{~g}$ tetra- $n$-butylammonium fluoride ( $1 \mathrm{mmol}, 10$ equiv.) was added, and the reaction mixture was stirred at room temperature until full conversion checked by TLC and HPLC-MS. The reaction mixture was concentrated and purified by preparative TLC plate with dichloromethane $\left(\mathrm{R}_{\mathrm{f}}=0.37\right)$.

Yield: $0.010 \mathrm{~g}(26 \%)$, yellow crystals, m.p. $271-272{ }^{\circ} \mathrm{C}$ (dichloromethane) ${ }^{1}{ }^{\mathrm{H}} \mathrm{NMR}(300 \mathrm{MHz}$, DMSO- $\left._{6}\right) \delta 10.19(\mathrm{~s}, 1 \mathrm{H}, \mathrm{OH}), 8.01(\mathrm{~d}, J=8.7 \mathrm{~Hz}, 1 \mathrm{H}, \mathrm{ArH}), 7.93(\mathrm{~d}, J=8.3 \mathrm{~Hz}, 2 \mathrm{H}, \operatorname{ArH}), 6.87(\mathrm{~d}$, $J=8.3 \mathrm{~Hz}, 2 \mathrm{H}, \mathrm{ArH}), 6.75(\mathrm{~s}, 1 \mathrm{H}, \mathrm{ArH}), 6.56(\mathrm{~d}, J=8.5 \mathrm{~Hz}, 1 \mathrm{H}, \mathrm{ArH}), 6.51(\mathrm{~s}, 1 \mathrm{H}, \mathrm{HC}=), 3.60-3.50(\mathrm{~m}$, $\left.2 \mathrm{H}, \mathrm{CH}_{2}\right), 3.42-3.33\left(\mathrm{~m}, 4 \mathrm{H}, \mathrm{CH}_{2}\right.$, overlap with water), $2.91-2.82\left(\mathrm{~s}, 2 \mathrm{H}, \mathrm{CH}_{2}\right), 2.04-1.93(\mathrm{~m}, 4 \mathrm{H}$, $\left.\mathrm{CH}_{2}\right)$ ppm; ${ }^{13} \mathrm{C}$ NMR $\left(125 \mathrm{MHz}, \mathrm{CDCl}_{3}\right) \delta 166.8(\mathrm{C}=\mathrm{O}), 161.6(\mathrm{C}=), 161.2(\mathrm{C}=), 151.4(\mathrm{C}=), 140.0(\mathrm{C}=)$, 130.6 $(\mathrm{C}=), 129.3(=\mathrm{CH}), 125.1(\mathrm{C}=), 115.9(=\mathrm{CH}), 113.7(\mathrm{C}=), 110.9(=\mathrm{CH}), 110.7(=\mathrm{CH}), 88.4(=\mathrm{CH}), 47.9$ $\left(\mathrm{CH}_{2}\right), 40.3\left(\mathrm{CH}_{2}\right.$, overlap with DMSO- $\left.d_{6}\right), 27.6\left(\mathrm{CH}_{2}\right), 25.4\left(\mathrm{CH}_{2}\right) \mathrm{ppm} ;{ }^{19} \mathrm{~F}$ NMR $\left(282 \mathrm{MHz}, \mathrm{CDCl}_{3}\right) \delta$ $-137.18(\mathrm{~d}, J=16.0 \mathrm{~Hz}, 1 \mathrm{~F}, \mathrm{BF}),-137.32(\mathrm{~d}, J=13.3 \mathrm{~Hz}, 1 \mathrm{~F}, \mathrm{BF}) \mathrm{ppm} ;[\mathrm{M}-\mathrm{F}]^{+}{ }_{\text {measured }}=363.1683$, calcd. for $\mathrm{C}_{21} \mathrm{H}_{21} \mathrm{BN}_{2} \mathrm{O}_{2} \mathrm{~F}: 363.1674$.

\section{Results and Discussion}

The common intermediate for the two designed chemodosimeters was planned to be 6-fluoro-1-methyl-3, 4-dihydroisoquinoline (9) that has been synthesized by following literature procedures [20]. 3-Fluorophenyl ethaneamine was acetylated, and a modified Bishler-Napieralski reaction resulted in compound 7 with good yields. The fluorinated isoquinoline (7) was then transformed to 6-pyrrolidinyl-1-methyl-3, 4-dihydroisoquinoline (9) by an aromatic nucleophilic substitution with pyrrolidine followed by an LDA (lithium diisopropylamide)-promoted acylation of the methyl group resulting in 1-(4-((tert-butyldiphenylsilyl) oxy) phenyl)-2-(6-(pyrrolidin-1-yl)-3, 4-dihydroisoquinolin-1 (2H)-ylidene) ethan-1-one (11a) and 1-(4-methoxyphenyl)-2-(6-(pyrrolidin-1-yl)-3, 4-dihydroisoquinolin-1 (2H)-ylidene) ethan-1-one (11b) with various yields. For this reaction step, an imine-enamine tautomerization is proposed involving the methyl group (see Supplementary Material). For the fluoride chemodosimeter 
(12a), the addition of the silylated benzoic acid ester (10a) was a straightforward approach, while, in the case of the $\mathrm{Pd}(0)$ sensing compound (12d), the allyloxycarbonyl group was not compatible with the strong basic conditions. Thus, at first, the methoxy group of $\mathbf{1 1 b}$ was demethylated with boron tribromide resulting in a phenolic compound 11c that was equipped by the allyloxycarbonyl recognition element in an acylation reaction with low yield. The reaction of 11a,d with boron trifluoride diethyl etherate in the presence of DIPEA (diisopropyl ethaneamine) was the final step resulting in the target compounds 1-(4-((tert-butyldiphenylsilyl) oxy) phenyl)-2-(2-(difluoroboranyl)-6-(pyrrolidin-1-yl)-3, 4-dihydroisoquinolin-1(2H)-ylidene) ethan-1-one (12a) and (Z)-allyl (4-(2-(2-(difluoroboranyl)-6-(pyrrolidin-1-yl)-3, 4-dihydroisoquinolin-1 (2H)-ylidene) acetyl) phenyl) carbonate (12d) (Scheme 1) with mediocre yields.

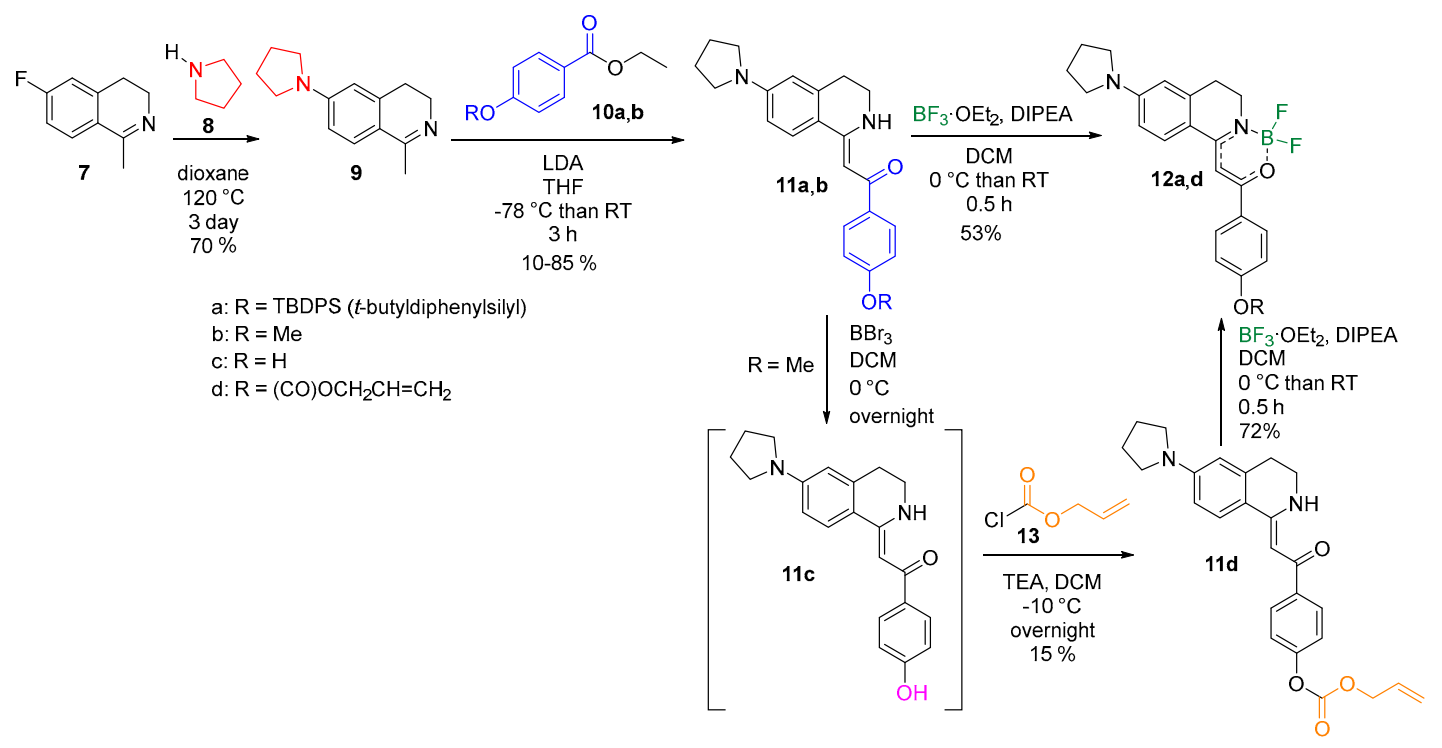

Scheme 1. The synthesis of the boroisoquinoline chemodosimeters.

After the successful synthesis of the two chemodosimeters (12a,d), we have investigated thoroughly their photophysical properties and their sensitivity and selectivity against the corresponding analytes. The photophysical properties were measured in acetonitrile (Table 1, Supplementary Figure S1). The molar absorbance coefficient corresponds to the absorbance maximum wavelength. One might conclude that $\mathbf{1 2 a}, \mathbf{d}$ has emission in the green range of the spectrum with a high Stokes-shift. Excellent quantum yield and acceptable $\varepsilon$ have been measured for 12a. However, although the quantum yield for $\mathbf{1 2 d}$ is low, with the high $\varepsilon$, it can still be considered as having appropriate brightness.

Table 1. Photophysical properties of the 12a,d measured in acetonitrile.

\begin{tabular}{ccccccc}
\hline Entry & Compound & $\lambda_{a b s}^{\max }(\mathbf{n m})$ & $\lambda_{\boldsymbol{e m}}^{\max }(\mathbf{n m})$ & $\boldsymbol{\Delta} \boldsymbol{\lambda}(\mathbf{n m})$ & $\mathcal{\varepsilon}\left(\mathbf{M}^{\mathbf{- 1}} \mathbf{c m}^{-\mathbf{1}}\right)$ & $\boldsymbol{\Phi}_{\boldsymbol{F}}$ \\
\hline 1 & 12a & 398 & 527 & 129 & 18,671 & 0.88 \\
2 & 12d & 408 & 532 & 124 & 82,101 & 0.10 \\
\hline
\end{tabular}

\subsection{The Fluoride Chemodosimeter}

Firstly, we have treated the solution of 12a in acetonitrile with one equivalent of crystalline tetrabutylammonium fluoride (TBAF. $3 \mathrm{H}_{2} \mathrm{O}$ ), and observed a decrease in the fluorescence intensity. Applying a larger amount of 10 equivalents fluoride completely turned off the fluorescence. Based on the basicity of the fluoride anion in organic solvents, it is proposed that, after the desilylation, a phenolate would be formed, but, in the presence of water, it is protonated leading to weak fluorescence. Applying a large excess of fluoride, the phenolate stays deprotonated. In this case, the negatively charged phenolate and its quinoidal mesomeric structure disrupt the conjugation of the phenyl group with 
the boranyl moiety leading to the loss of the emission. In order to prove this theory, we have isolated the desilylated phenolic compound (12c) and we have proven its structure by HPLC-MS and NMR techniques. 12c showed weak fluorescence, and, after the deprotonation with potassium tert-butylate, the emission of the substance was switched off. Notably, in the presence of dry TBAF in the absence of water, the emission was completely turned off as well (Scheme 2).
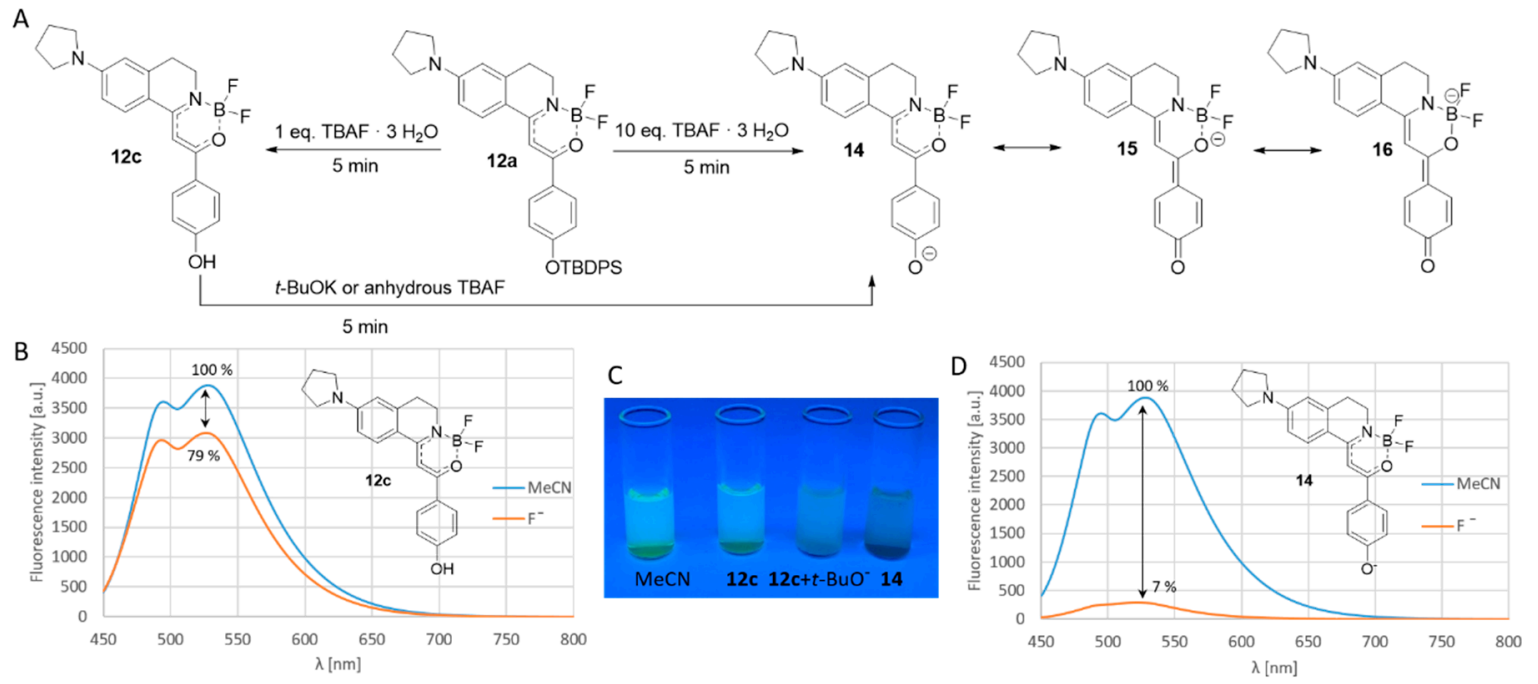

Scheme 2. (A) proposed mechanism of fluoride detection by 12a; (B) emission spectra of 12c; (C) visible changes in the reactions of 12a; (D) emission spectra of $\mathbf{1 4}$.

The anion-selectivity of compound 12a was tested with 100 equiv. tetrabutylammonium salts in acetonitrile. The applied anions were halogens $\left(\mathrm{Cl}^{-}, \mathrm{Br}^{-}, \mathrm{I}^{-}\right), \mathrm{HSO}_{4}^{-}, \mathrm{OAc}^{-}, \mathrm{OH}^{-}, \mathrm{SCN}^{-}, \mathrm{BF}_{4}^{-}, \mathrm{PF}_{6}^{-}$, $\mathrm{CN}^{-}, \mathrm{N}_{3}{ }^{-}, \mathrm{H}_{2} \mathrm{PO}_{4}^{-}$and $\mathrm{MeSO}_{3}{ }^{-}$. It was found that, besides the fluoride, the fluorescence intensity was turned off in the presence of $\mathrm{OH}^{-}$and decreased by $\mathrm{OAc}^{-}$(Figure 4). However, applying 10 equiv. excess of the anions, the selectivity was proven to be complete (Figures 4 and 5a); only the fluoride turned off the emission rapidly, while the effect of $\mathrm{OH}^{-}$and $\mathrm{AcO}^{-}$was not significant even after a long time (Figure 5b).

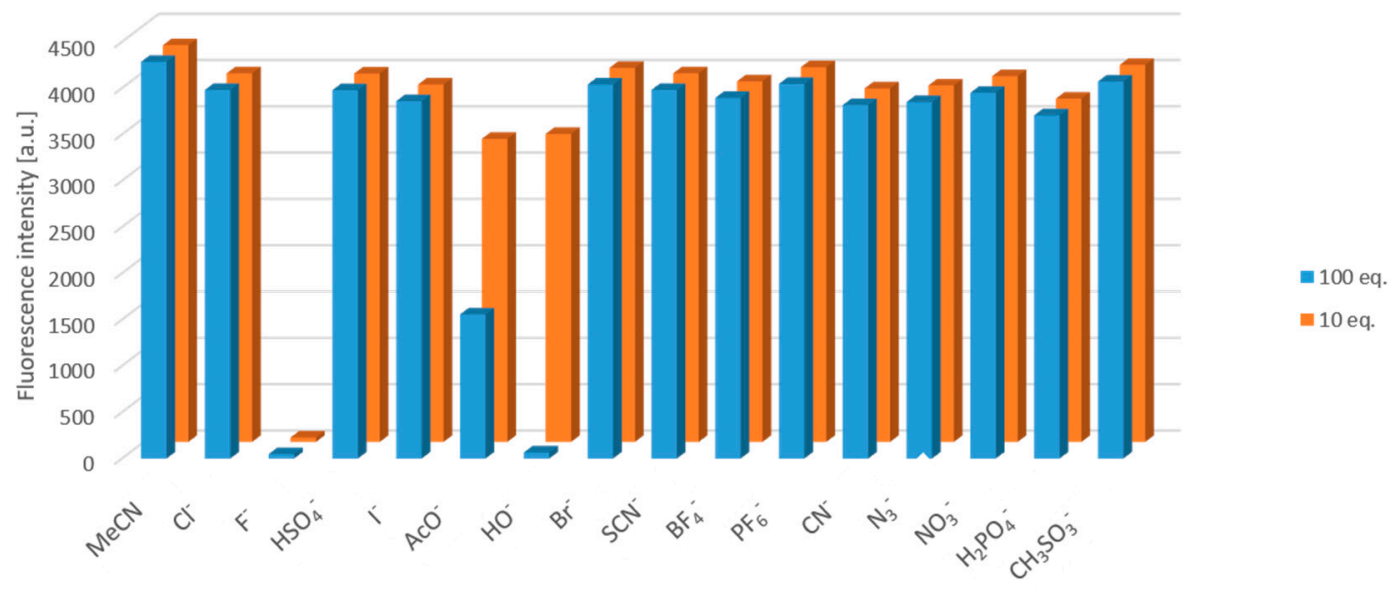

Figure 4. Fluorescence intensity changes caused by the anions at the wavelength of the maximal intensity after $5 \mathrm{~min}$. 
(a)

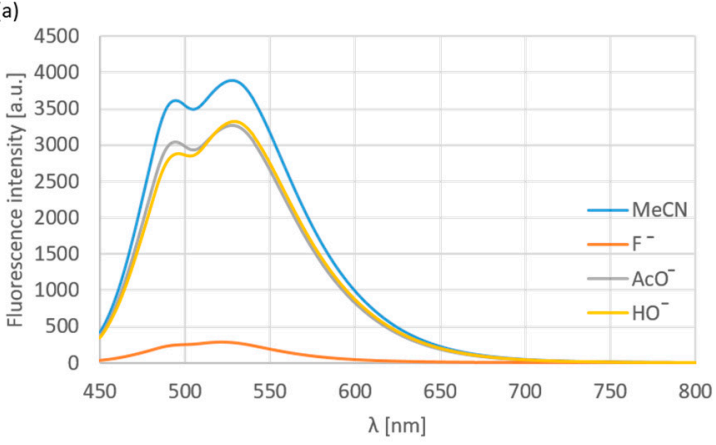

(b)

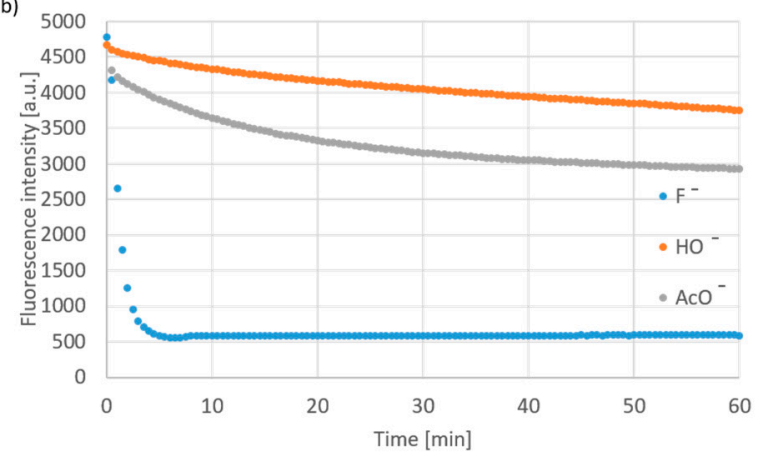

Figure 5. (a) fluorescence spectra obtained in the presence of 10 equivalents fluoride, acetate and hydroxy; (b) changes of the emission intensity during $60 \mathrm{~min}$ at $528 \mathrm{~nm}$ in the presence of the same anions.

The presence of fluoride shows an effect not only on the emission, but on the absorption as well. The effect is easily visible in the absorbance spectrum (Supplementary Figure S2) and with the naked eyes as well, which might be advantageous by field measurements and tests (Figure 6).

(a)

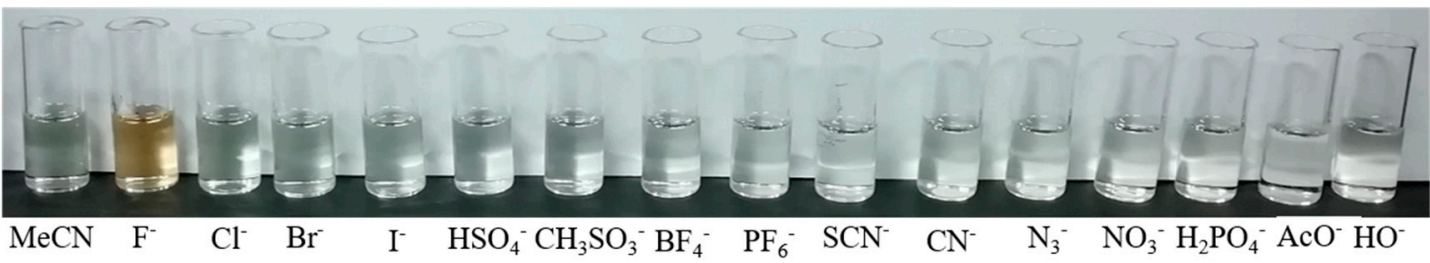

(b)

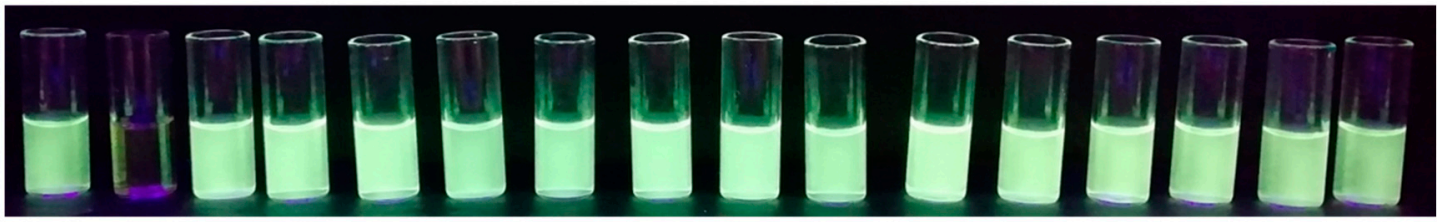

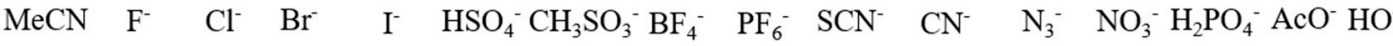

Figure 6. (a) photo of the chemodosimeter in acetonitrile with the anions without excitation; (b) photo of the chemodosimeter in acetonitrile with the anions applying excitation at $366 \mathrm{~nm}$.

A titration curve has been recorded with TBAF. $3 \mathrm{H}_{2} \mathrm{O}$ and the solution of chemodosimeter $(1 \mu \mathrm{M}$, 12a) (Figure 7). The fluorescence intensity decreased linearly until TBAF concentration of $3.5 \mu \mathrm{M}$, and thereafter the fluorescence turned off completely.

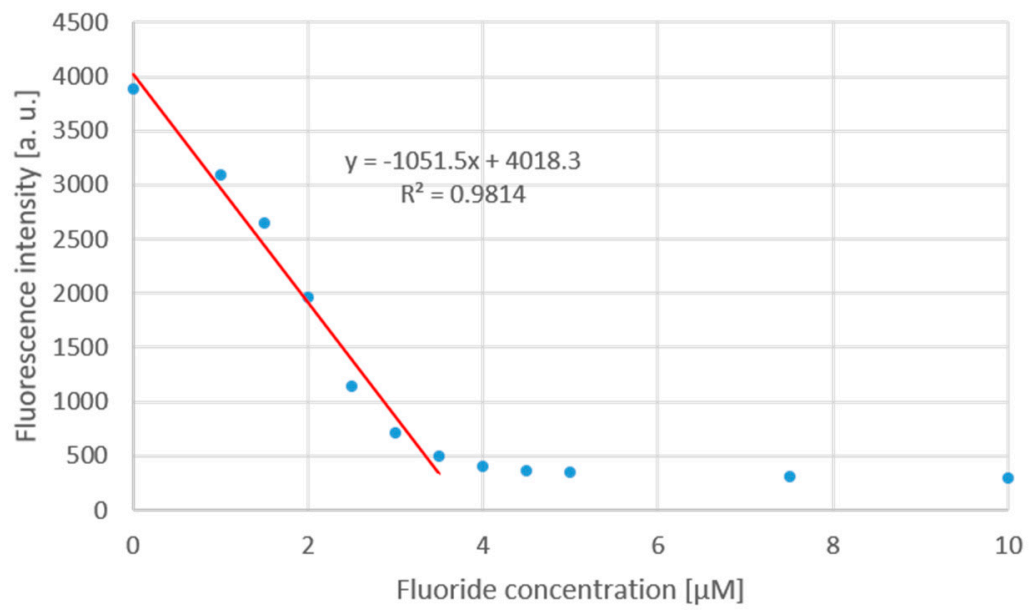

Figure 7. The loss of fluorescence intensity as a function of the fluoride concentration. 
Considering that the chemodosimeters are used for prompt measurements on the field for examining the quality of water (like open water sources, springs, wells, and waste water), it is necessary to check the efficacy of the 12a chemodosimeter in water solution. However, the boroisoquinolines underwent aggregation in pure water; therefore, $\mathrm{PBS}$ buffer $(50 \mathrm{mM}$ phosphate, $\mathrm{pH}=7.4)$ with $0.1 \%$ SDS was applied. It should be mentioned that investigating absorbance and fluorescence spectra of 12a in dimethyl formamide, dimethyl acetamide and DMSO, the absorbance and fluorescence intensity decreased to $\sim 40 \%$ (see Supplementary Figure S1), which can be addressed to the polarity of the media. The concentration of the chemodosimeter was set to $10 \mu \mathrm{M}$, and the change of the emission spectra was examined by different fluoride concentrations. Firstly, it was observed that the medium is significantly influencing the function of the chemodosimeter; in particular, in aqueous solution, the emission intensity of the fluorescent probe increased. Notably, this is more favorable than the switch-off mechanism. However, the emission maximum moved to the direction of blue light. It is supposed that, in water, the fluoride ion is a less strong base; furthermore, the phenolic OH-group can be protonated in the aqueous medium, eliminating the negative charge responsible for turning off the fluorescence. Although the addition of $0.1,1$ and 10 equivalents of the analyte did not cause significant emission growth over a 60-minute period, but 100 equivalents of the fluoride doubled the emission intensity at the maximum wavelength after 5 min (Figure 8).

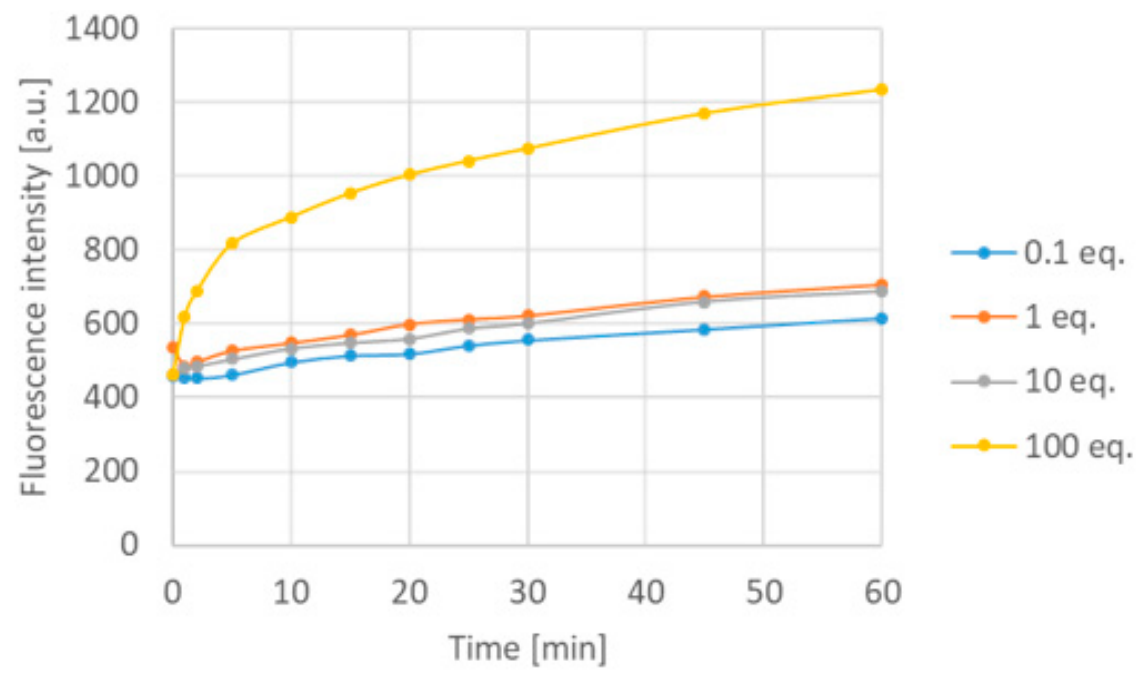

Figure 8. Time-dependency of the emission of 12a in aqueous solution at $489 \mathrm{~nm}$.

\subsection{The Pd (0) Chemodosimeter}

The same methodology was followed in this case as that for the fluoride chemodosimeter. We have treated compound $\mathbf{1 2 d}$ with 100 equiv. of metals or metal cations besides $\mathrm{Pd}_{2} \mathrm{dba}_{3}$ [tris(dibenzylideneacetone)dipalladium], in particular $\mathrm{Ag}^{+}, \mathrm{Ca}^{2+}, \mathrm{Cu}^{2+}, \mathrm{Fe}^{2+}, \mathrm{Fe}^{3+}, \mathrm{Hg}^{2+}, \mathrm{Mg}^{2+}$, $\mathrm{Mn}^{2+}, \mathrm{Ni}^{2+}, \mathrm{Pb}^{2+}, \mathrm{Pt}^{2+}, \mathrm{Ru}^{3+}, \mathrm{Zn}^{2+}$ (Figure 9). In most cases, chloride salts were available, except of $\mathrm{Hg}$ and $\mathrm{Pb}$, which were used as an iodide and nitrate salt, respectively. In general, there were no spectral changes to observe, only in the case of $\mathrm{Pd}(0)$ that decreased the intensity and shifted the emission maximum to a lower range (from $532 \mathrm{~nm}$ to $491 \mathrm{~nm}$ ). However, by several other metal ions $\left(\mathrm{Ru}^{3+}, \mathrm{Cu}^{2+}, \mathrm{Pt}^{2+}, \mathrm{Fe}^{3+}\right)$, the decrease of the intensity was observed as well, but there was no shift. This effect was caused by the absorption of the exciting light by the transition metals [21,22]. Based on HPLC-MS measurements, it has been proven that no chemical reaction occurred in the presence of the latter cations. On the contrary, $\mathrm{Pd}(0)$ caused the leave of the allyloxy group with full conversion, resulting in compound $12 \mathrm{c}$ together with turning off the emission. 


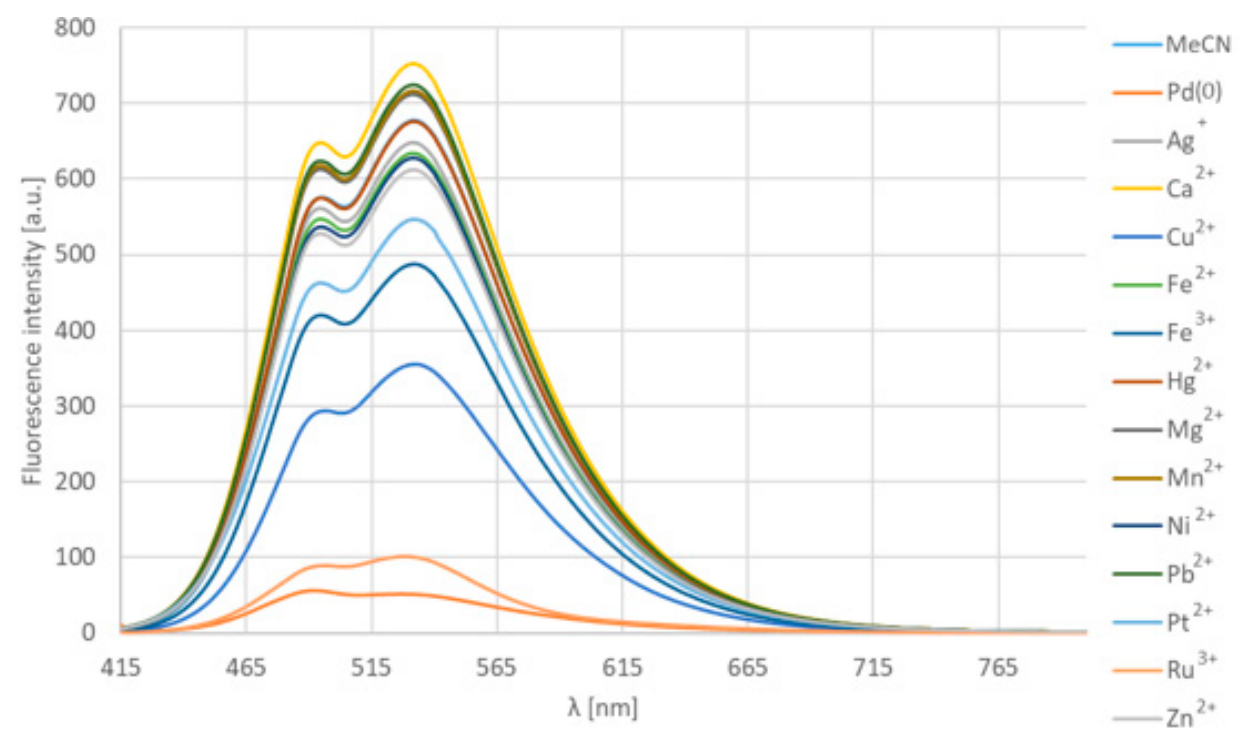

Figure 9. The emission spectra of $\mathbf{1 2 d}$ in the presence of 100 equivalents of metals and different cations in acetonitrile at room temperature after $5 \mathrm{~min}$.

The mechanism of action of $\mathrm{Pd}(0)$ with allyloxy recognition functional groups was proposed in numerous earlier cases similarly [17], thus it might be accepted that firstly the $\mathrm{Pd}(0)$ metal associates with the electron system of the allyloxy group cleaving the $\mathrm{O}-\mathrm{C}$ bond resulting in a propylene released. Meanwhile, $\mathrm{Pd}(0)$ turns to its oxidized form Pd (II). After the dissociation of the metal, a $\mathrm{CO}_{2}$ molecule is able to leave intermediate $\mathbf{1 8}$ by decarboxylation resulting in compound $\mathbf{1 2 c}$ (Scheme 3).

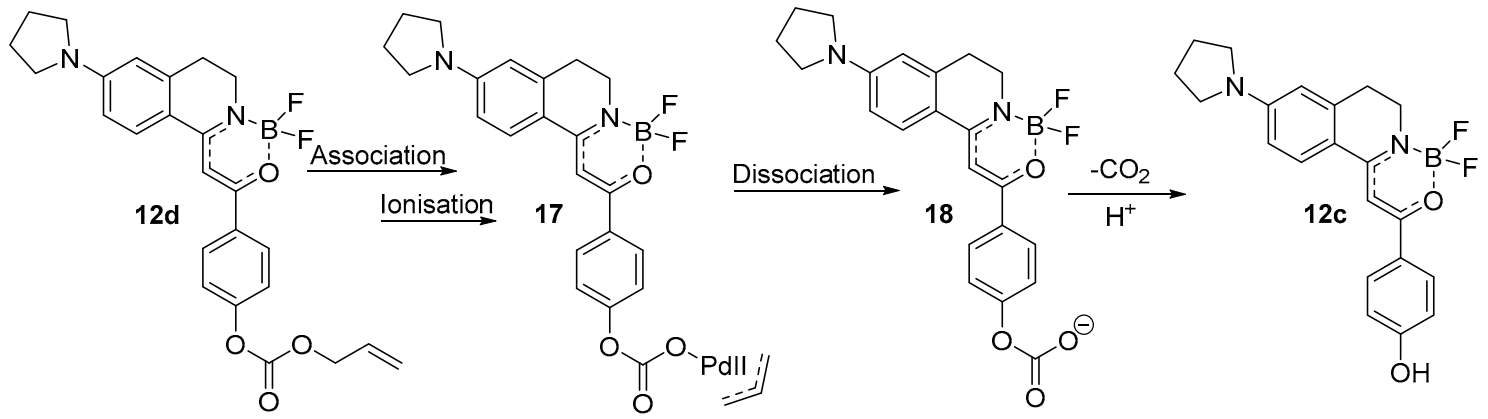

Scheme 3. The supposed mechanism for the Pd (0) detection of $\mathbf{1 2 d}$.

Further examination of the selectivity with different palladium salts and $\mathrm{Pd}(0)$ dibenzylideneacetone-complex was accomplished (Figure 10). Moreover, Ni (0) and Pt (0) was added to these confirmative experiments. One might observe that substance 12d is not sensible for $\mathrm{Pd}$ (II) salts $\left(\mathrm{PdCl}_{2}, \mathrm{Pd}(\mathrm{OAc})_{2}\right)$, but selectively, only for the Pd (0) complex. The addition of $\mathrm{Ni}(0)$ and $\mathrm{Pt}(0)$ caused a significant drop in the fluorescence, but no change in the shape of the spectrum. Notably, MS measurements have proven that $\mathrm{Pd}_{2} \mathrm{dba}_{3}$ was the only agent causing the chemical reaction responsible for the considerable change in the emission spectrum. Ten equivalents of the metals and salts were used with an incubation time of $5 \mathrm{~min}$. The observed intensity loss is associated with the light absorption of the metal. 


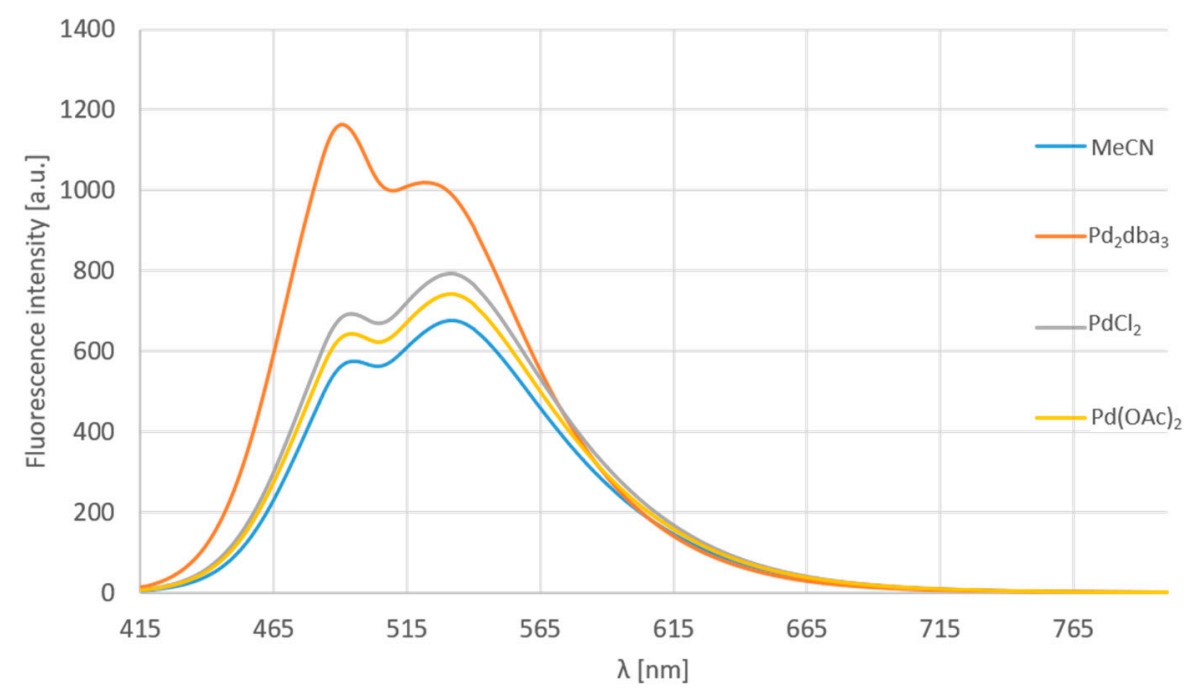

Figure 10. The emission spectra of $\mathbf{1 2 d}$ in the presence of 10 equivalent different palladium compounds.

The titration of the acetonitrile solution of compound $\mathbf{1 2 d}$ with $\mathrm{Pd}_{2} \mathrm{dba}_{3}$ (Figure 11) showed that, until four equivalents the intensity grows, while, applying a larger excess, it decreases possibly due to the absorption of the light by the metal in high concentration.

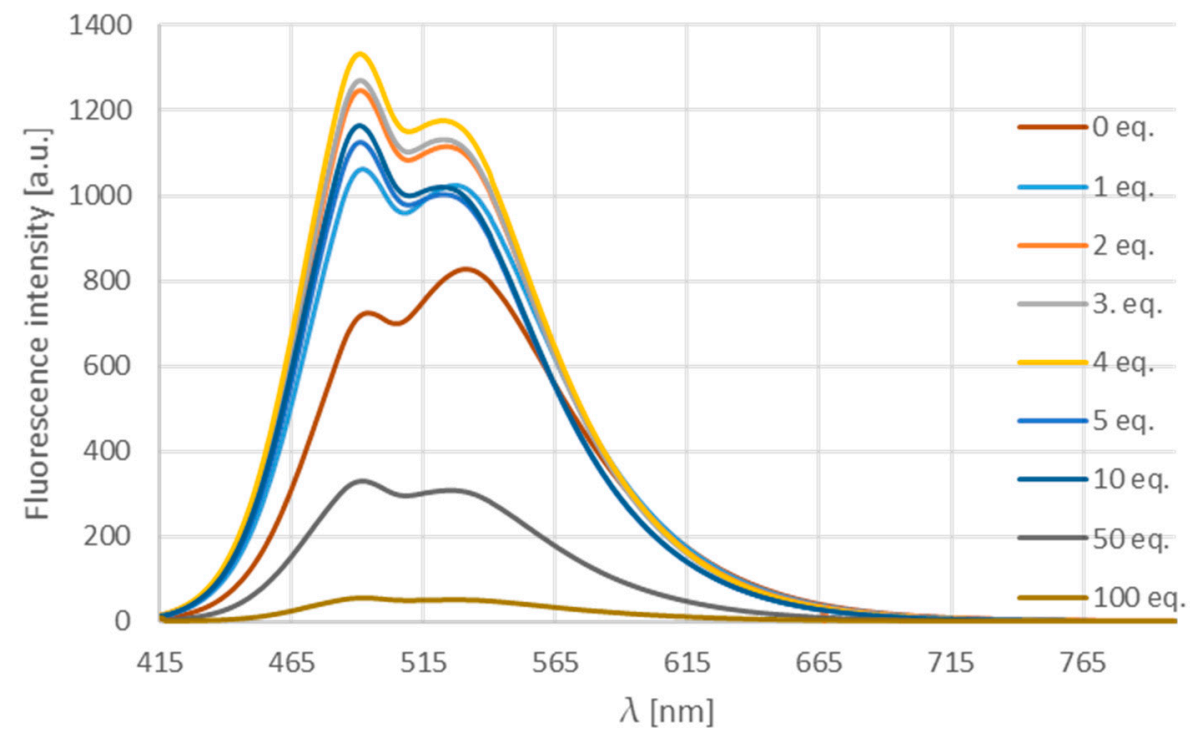

Figure 11. The emission spectra of $\mathbf{1 2 d}$ in the presence of different equivalents $\operatorname{Pd}_{2} \mathrm{dba}_{3}$.

Investigating absorbance and fluorescence spectra of $\mathbf{1 2 d}$ in polar solvents, in particular, dimethyl formamide, dimethyl acetamide and DMSO, the absorbance and fluorescence intensity did not show any change (see Supplementary Figure S3). The emission spectrum was measured in the presence of $\mathrm{Pd}_{2} \mathrm{dba}_{3}$ in aqueous solution as well. In this case, the metal light absorption was similarly significant at 10 equivalents excess; therefore, the amount of $\mathrm{Pd}_{2} \mathrm{dba}_{3}$ was decreased to $10 \%$ or 1 equivalent resulting in an increase in the fluorescence after $5 \mathrm{~min}$ and forming a saturation curve in both cases (Figure 12). 


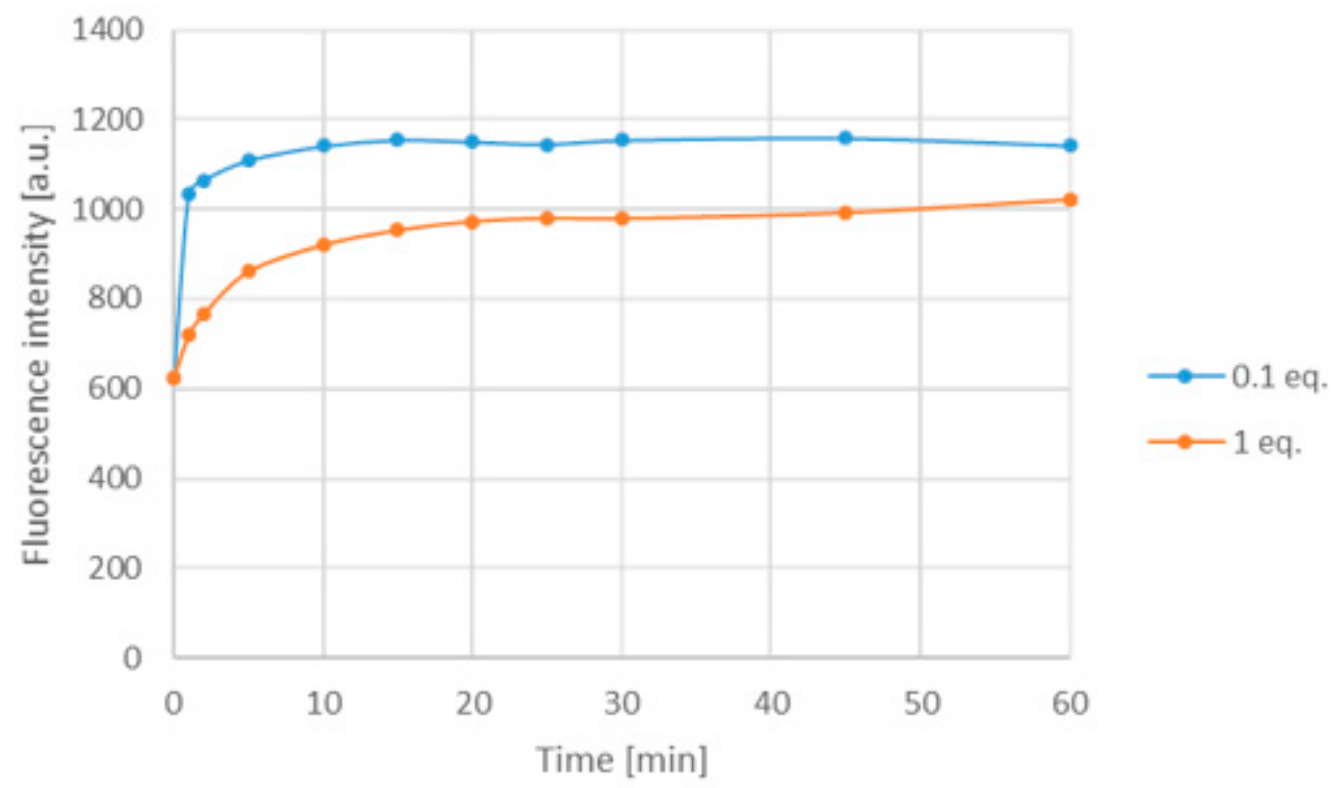

Figure 12. The change of the emission of $\mathbf{1 2 d}$ in water at $537 \mathrm{~nm}$.

\section{Conclusions}

Based on the development of a family of new fluorophores, we have successfully applied appropriately substituted derivatives on the field of analytical detection. New fluoride- and Pd (0)-sensing molecules were synthesized with good overall yields in multistep syntheses. Both chemodosimeters showed excellent selectivity toward the corresponding analyte. The fluoride-selective boroisoquinoline showed some restriction on the concentration, while the other one was able to detect $\mathrm{Pd}(0)$ selectively towards Pd with other oxidation state even at catalytic concentration. Moreover, the boroisoquinoline chemodosimeters were successfully applied in aqueous media as well extending the possible application fields.

Supplementary Materials: The following are available online at http://www.mdpi.com/1996-1944/13/1/199/s1, synthesis methods for compounds known in the literature, absorbance, excitation and emission spectra, ${ }^{1} \mathrm{H}$ and ${ }^{13}$ C spectra.

Author Contributions: Conceptualization, P.Á.-B.; methodology, P.Á.-B. and D.S.; resources, G.M.K.; syntheses and characterization, D.S.; writing—original draft preparation, D.S.; writing—review and editing, P.Á.-B.; funding acquisition, P.Á.-B. and G.M.K. All authors have read and agreed to the published version of the manuscript.

Funding: This research was funded by National Research, Development and Innovation Office, Grant No. 124598.

Acknowledgments: The authors acknowledge the help and advices of Attila Kormos.

Conflicts of Interest: The authors declare no conflict of interest.

\section{References}

1. Jiao, Y.; Zhu, B.; Chen, J.; Duan, X. Fluorescent sensing of fluoride in cellular system. Theranostics 2015, 5, 173-187. [CrossRef]

2. Kielhorn, J.; Melber, C.; Keller, D.; Mangelsdorf, I. Palladium-A review of exposure and effects to human health. Int. J. Hyg. Environ. Health 2002, 205, 417-432. [CrossRef]

3. Kolemen, S.; Akkaya, E.U. Reaction-based BODIPY probes for selective bio-imaging. Coord. Chem. Rev. 2018, 354, 121-134. [CrossRef]

4. Chen, P.; Bai, W.; Bao, Y. Fluorescent chemodosimeters for fluoride ions via silicon-fluorine chemistry: 20 years of progress. J. Mater. Chem. C 2019, 7, 11731-11746. [CrossRef] 
5. Yamaguchi, S.; Akiyama, S.; Tamao, K. Photophysical properties changes caused by hypercoordination of organosilicon compounds: From trianthrylfluorosilane to trianthryldifluorosilicate. J. Am. Chem. Soc. 2000, 122, 6793-6794. [CrossRef]

6. Song, F.; Garner, A.L.; Koide, K. A highly sensitive fluorescent sensor for palladium based on the allylic oxidative insertion mechanism. J. Am. Chem. Soc. 2007, 129, 12354-12355. [CrossRef] [PubMed]

7. Kaur, K.; Saini, R.; Kumar, A.; Luxami, V.; Kaur, N.; Singh, P.; Kumar, S. Chemodosimeters: An approach for detection and estimation of biologically and medically relevant metal ions, anions and thiols. Coord. Chem. Rev. 2012, 256, 1992-2028. [CrossRef]

8. Izuora, K.; Twombly, J.G.; Whitford, G.M.; Demertzis, J.; Pacifici, R.; Whyte, M.P. Skeletal fluorosis from brewed tea. J. Clin. Endocrinol. Metab. 2011, 96, 2318-2324. [CrossRef]

9. Mohammadi, A.A.; Yousefi, M.; Yaseri, M.; Jalilzadeh, M.; Mahvi, A.H. Skeletal fluorosis in relation to drinking water in rural areas of West Azerbaijan, Iran. Sci. Rep. 2017, 7, 17300. [CrossRef]

10. Kakumanu, N.; Rao, S.D. Skeletal fluorosis due to excessive tea drinking. N. Engl. J. Med. 2013, $368,1140$. [CrossRef]

11. Liu, B.; Tian, H. A ratiometric fluorescent chemosensor for fluoride ions based on a proton transfer signaling mechanism. J. Mater. Chem. 2005, 15, 2681-2686. [CrossRef]

12. Zheng, X.; Zhu, W.; Liu, D.; Ai, H.; Huang, Y.; Lu, Z. Highly selective colorimetric/fluorometric dual-channel fluoride ion probe, and its capability of differentiating cancer cells. ACS Appl. Mater. Interfaces 2014, 6, 7996-8000. [CrossRef] [PubMed]

13. Zhang, Q.; Piao, X.L.; Piao, X.S.; Lu, T.; Wang, D.; Kim, S.W. Preventive effect of Coptis chinensis and berberine on intestinal injury in rats challenged with lipopolysaccharides. Food Chem. Toxicol. 2011, 49, 61-69. [CrossRef] [PubMed]

14. Elsayed, S.; Agostini, A.; Santos-Figueroa, L.E.; Martínez-Máñez, R.; Sancenón, F. An instantaneous and highly selective chromofluorogenic chemodosimeter for fluoride anion detection in pure water. ChemistryOpen 2013, 2, 58-62. [CrossRef]

15. Cao, J.; Zhao, C.; Feng, P.; Zhang, Y.; Zhu, W. A colorimetric and ratiometric NIR fluorescent turn-on fluoride chemodosimeter based on BODIPY derivatives: High selectivity via specific Si-O cleavage. RSC Adv. 2012, 2, 418-420. [CrossRef]

16. Kim, S.Y.; Hong, J.I. Chromogenic and fluorescent chemodosimeter for detection of fluoride in aqueous solution. Org. Lett. 2007, 9, 3109-3112. [CrossRef]

17. Wang, P.; Xia, J.; Gu, Y. A novel NIR fluorescent probe for palladium detection based on $\mathrm{Pd}(0)$ mediated reaction. Tetrahedron Lett. 2015, 56, 6491-6494. [CrossRef]

18. Chen, Y.; Chen, B.; Luo, D.; Cai, Y.; Wei, Y.; Han, Y. A facile naphthalene-based fluorescent "turn-on" chemodosimeter for palladium ions in aqueous solution. Tetrahedron Lett. 2016, 57, 1192-1195. [CrossRef]

19. Huang, J.; Ding, Y.; Fu, H.; Chen, B.; Han, Y. A water soluble ESIPT-based fluorescent chemodosimeter for the ratiometric detection of palladium ions in aqueous solution and its application in live-cell imaging. N. J. Chem. 2018, 42, 15587-15592. [CrossRef]

20. Sóvári, D.; Kormos, A.; Demeter, O.; Dancsó, A.; Keserû, G.M.; Milen, M.; Ábrányi-Balogh, P. Synthesis and fluorescent properties of boroisoquinolines, a new family of fluorophores. RSC Adv. 2018, 8, 38598-38605. [CrossRef]

21. Kettle, S.F.A. Electronic spectra of transition metal complexes. In Physical Inorganic Chemistry; Springer: Berlin/Heidelberg, Germany, 1996; pp. 156-184.

22. Hao, Y.; Chen, S.; Zhou, Y.; Zhang, Y.; Xu, M. Recent Progress in Metal-Organic Framework (MOF) Based Luminescent Chemodosimeters. Nanomaterials 2019, 9, 974. [CrossRef] [PubMed]

(C) 2020 by the authors. Licensee MDPI, Basel, Switzerland. This article is an open access article distributed under the terms and conditions of the Creative Commons Attribution (CC BY) license (http://creativecommons.org/licenses/by/4.0/). 